OPEN ACCESS

Edited by:

Yih-Kuen Jan,

University of llinois at

Urbana-Champaign, United States

Reviewed by:

Thomas Seel,

Technische Universität Berlin,

Germany

Qichang Mei,

University of Auckland, New Zealand

*Correspondence:

Mathieu Falbriard

mathieu.falbriard@epfl.ch

Specialty section:

This article was submitted to Biomechanics,

a section of the journal Frontiers in Bioengineering and Biotechnology

Received: 07 November 2019

Accepted: 27 January 2020

Published: 13 February 2020

Citation:

Falbriard M, Meyer F, Mariani B, Millet GP and Aminian K (2020) Drift-Free Foot Orientation Estimation

in Running Using Wearable IMU.

Front. Bioeng. Biotechnol. 8:65.

doi: 10.3389/fbioe.2020.00065

\section{Drift-Free Foot Orientation Estimation in Running Using Wearable IMU}

\author{
Mathieu Falbriard ${ }^{1 *}$, Frédéric Meyer ${ }^{2}$, Benoît Mariani3 ${ }^{3}$, Grégoire P. Millet ${ }^{2}$ and \\ Kamiar Aminian ${ }^{1}$
}

${ }^{1}$ Laboratory of Movement Analysis and Measurement, EPFL, Lausanne, Switzerland, ${ }^{2}$ Institute of Sport Sciences, University of Lausanne, Lausanne, Switzerland, ${ }^{3}$ Gait Up S.A., Lausanne, Switzerland

This study aimed to introduce and validate a new method to estimate and correct the orientation drift measured from foot-worn inertial sensors. A modified strap-down integration (MSDI) was proposed to decrease the orientation drift, which, in turn, was further compensated by estimation of the joint center acceleration (JCA) of a twosegment model of the foot. This method was designed to fit the different foot strike patterns observed in running and was validated against an optical motion-tracking system during level treadmill running at 8,12 , and $16 \mathrm{~km} / \mathrm{h}$. The sagittal and frontal plane angles obtained from the inertial sensors and the motion tracking system were compared at different moments of the ground contact phase. The results obtained from 26 runners showed that the foot orientation at mean stance was estimated with an accuracy (inter-trial median \pm IQR) of $0.4 \pm 3.8^{\circ}$ and a precision (inter-trial precision median \pm IQR) of $3.0 \pm 1.8^{\circ}$. The orientation of the foot shortly before initial contact (IC) was estimated with an accuracy of $2.0 \pm 5.9^{\circ}$ and a precision of $1.6 \pm 1.1^{\circ}$; which is more accurate than commonly used zero-velocity update methods derived from gait analysis and not explicitly designed for running. Finally, the study presented the effect initial and terminal contact (TC) detection errors have on the orientation parameters reported.

Keywords: running, inertial measurement units, validation study, orientation, drift, angles, foot strike

\section{INTRODUCTION}

The orientation of the foot recorded slightly before, during, or after the ground contact phase is an essential parameter for running analysis. Many studies have investigated how different foot landing techniques give rise to kinematic and kinetic differences between subjects. For instance, the foot strike patterns (i.e., rearfoot, midfoot, and forefoot) and their association with injury risks (Cavanagh and Lafortune, 1980; Daoud et al., 2012; Goss and Gross, 2012), running economy (Perl et al., 2012; Gruber et al., 2013; Miller and Hamill, 2015; Hamill and Gruber, 2017), running performance (Larson et al., 2011; Kasmer et al., 2013; de Almeida et al., 2015), collision forces (Lieberman et al., 2010; Boyer et al., 2014; Gruber et al., 2014), muscle activity (Ahn et al., 2014; Yong et al., 2014), and footwear (Lorenz and Pontillo, 2012; Horvais and Samozino, 2013; Larson, 2014; Meyer et al., 2018) have been at the core of many research studies and changes of running paradigms within the last decades. The orientation of the foot in different planes or relative to the 
shank has also been extensively analyzed and now constitutes a primary marketing argument for the running industry (e.g., eversion/inversion and pronation/supination) (Nigg et al., 1993; Perry and Lafortune, 1995; Monaghan et al., 2014; Muñoz-Jimenez et al., 2015).

In research, the continuous measurement of the $3 \mathrm{D}$ orientation of the foot is generally obtained using optical motion capture systems (Arndt et al., 2007; Riley et al., 2008; Altman and Davis, 2012). While these systems measure the foot pose (i.e., orientation and position) accurately, they are often restricted to well-equipped laboratories and treadmill running. As an alternative to this lack of portability, a growing number of studies have shown that wearable inertial sensors, if combined with state-of-the-art algorithms, can be used to provide reliable spatiotemporal information (Camomilla et al., 2018).

Historically, the methods based on foot-worn inertial sensors that estimate the fixed-frame orientation of the foot first emerged from the field of gait analysis. Although different methods have been proposed (Sabatini et al., 2005; Mariani et al., 2010; Skog et al., 2010), most share the same underlying structure: (1) integration of the angular velocity obtained from a foot-mounted gyroscope to calculate the global frame (GF) orientation and (2) combine the measurements from other sensors (e.g., accelerometer, magnetometer, and GPS) to estimate and remove the orientation drift. Methods such as the zerovelocity-update usually require the presence of low accelerations or low magnetic disturbances during the period of stance to estimate the orientation drift. Although these periods are generally present during low-speed human locomotion, they are either rare or inexistent as the speed increases (Foxlin, 2005; Park and Suh, 2010; Zhang et al., 2017), and thus are likely to underperform in running.

Nevertheless, studies have proposed a hard reset of the drift based on the hypothetical presence of a foot-flat period during the stance phase of running (Bailey and Harle, 2014; Chew et al., 2018; Yuan et al., 2019). Although this approach seems reasonable for rearfoot strikers, it is not appropriate for forefoot strikers as their rearfoot segment possibly never comes into contact with the ground. Also, typically rearfoot strikers tend to switch from a rearfoot to a forefoot strike pattern when the running speed increases (Breine et al., 2014); speed might likewise be a confounding factor for any drift reduction method. Note that if the continuous orientation is not required, different approaches have been proposed to classify the foot-strike patterns with footworn inertial measurement units (IMUs) (Strohrmann et al., 2011; Giandolini et al., 2014).

The combination of strap-down integration with the difference between proximal and distal accelerations at any joint center has been used to estimate the joint orientation and to model the drift in dynamic movements (Dejnabadi et al., 2006; Fasel et al., 2018). To the authors' knowledge, this method has never been tested to estimate the orientation drift of the foot in running.

Hence, the objective of this research was to propose a novel drift-free orientation estimation method for running built on a two-segment model of the foot and explore the abovementioned combination of proximal and distal accelerations using a single
IMU placed on the rear foot. We assumed that, regardless of the foot strike pattern, a forefoot-flat period is always present, and it can be used to estimate and compensate the foot orientation drift. The proposed method provides an estimate of the orientation drift for each stance period and can, therefore, be used for online analysis of the running gait. Moreover, the proposed method does not require the presence of a second IMU on the forefoot, for such complicated instrumentation would reduce its applicability for field studies.

\section{MATERIALS AND METHODS}

\section{Protocol}

A total of 26 volunteers ( 9 females and 17 males, age $29 \pm 6$ years, weight $70 \pm 10 \mathrm{~kg}$, height $174 \pm 8 \mathrm{~cm}$, running weekly $2.1 \pm 1.0 \mathrm{~h}$, 11 affiliated to a running club) participated in this study. They were running at least once a week and had no symptomatic musculoskeletal injuries. Participants gave their written informed consent before the measurements and ran for $45 \mathrm{~s}$ at 8,12 , and $16 \mathrm{~km} / \mathrm{h}$ on a level instrumented treadmill, wearing their regular shoes. A 6 min familiarization period (Lavcanska et al., 2005) was performed on the treadmill and served as a warm-up for the participants. This protocol was approved by the local ethical committee (CCER-VD 2015-00006) and conducted according to the declaration of Helsinki.

\section{Wearable Systems Inertial Measurement Units}

One Inertial Measurement Unit (Physilog ${ }^{\circledR}$ 4, GaitUp SA, CH, weight: 19 g, size: $50 \mathrm{~mm} \times 37 \mathrm{~mm} \times 9.2 \mathrm{~mm}$ ) was fixed on the dorsum of each foot using a Silicon/Velcro elastic strap. The accelerometer operated at $500 \mathrm{~Hz}( \pm 16 \mathrm{~g})$, the gyroscope at $500 \mathrm{~Hz}( \pm 2000 \mathrm{deg} / \mathrm{s})$, and sensors' calibration was performed according to Ferraris et al. (1995). We modeled the foot with two rigid segments: the rearfoot and forefoot segments (Figure 1). Note that there was no sensor located on the forefoot segment. We aligned the IMU's technical frame (TF) with the rearfoot functional frame $\left(\mathrm{FF}_{\text {rear }}\right)$, as described by Falbriard et al. (2018); we recorded a standing period and used the gravitational acceleration to set the $\mathrm{FF}_{\text {rear }} y$-axis parallel to the vertical axis of the foot. Then, using principal component analysis (PCA) on the running measurements, we aligned the $\mathrm{FF}_{\text {rear }} z$-axis with the principal vector, which we assumed parallel to the mediolateral axis of the foot. Finally, we defined the $\mathrm{FF}_{\text {rear }} x$-axis as the cross-product between the $\mathrm{FF}_{\text {rear }} y$-axis and the $z$-axis. Note that the calibration matrix was considered constant within the duration of the trial.

\section{Temporal Events Detection}

Temporal events detection was based on previously validated algorithms (Falbriard et al., 2018). We segmented the trials into running strides and extracted four events per stance phase. The Initial (IC) and Terminal (TC) contact events, when the foot initializes and terminates ground contact, were found using local minima on the pitch angular velocity. Also, the mean-stance (MS) was defined as the mean time between IC and TC, and MinRot 


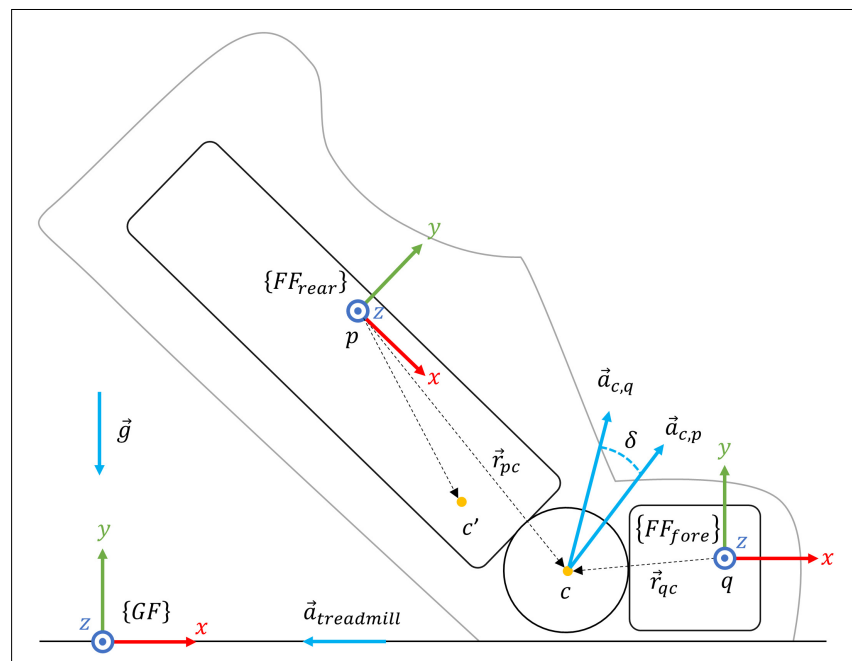

FIGURE 1 | The two-segments model of the foot during the stance phase. Using the RGB convention, $\left\{F F_{\text {rear }}\right\}$ represents the FF of the rearfoot segment, $\left\{F F_{\text {fore }}\right\}$ the $F F$ of the forefoot segment, and $\{G F\}$ the room's global frame. Points $p$ and $q$ are arbitrarily placed on rearfoot, and forefoot segments, $c^{\prime}$ and $c$ are, respectively, hypothetic and optimum rearfoot-forefoot joint's center. $\vec{a}_{c, q}$ is the acceleration at c estimated from $q, \vec{a}_{c, p}$ the acceleration at $c$ estimated from $p, \vec{a}_{\text {treadmill }}$ the acceleration of the treadmill, and $\vec{g}$ the Earth gravitational acceleration. Finally, $\delta$ is the orientation difference (i.e., quaternion) between $\vec{a}_{c, p}$ and $\vec{a}_{c, q}$ while $\vec{r}_{p c}$ and $\vec{r}_{q c}$ are the distance vectors from point $p$ to $c$ and from $q$ to $c$, respectively.

as the time-point of stance when the norm of the foot angular velocity is minimum.

\section{Orientation Estimation}

Strap-down integration of the angular velocity (Favre et al., 2008) is frequently used to obtain the orientation of a body segment in the GF. However, this operation generates a drift which accumulates with time. In this study, orientation estimation was performed in three phases: (i) modified strap-down integration (MSDI), (ii) drift modeling, and (iii) drift estimation and reduction. The MSDI method provides a first estimate of the orientation. It assumes that, at MinRot of each stance phase, the $\mathrm{FF}_{\text {rear }}$ and the GF are aligned. In other words, it supposes that a rearfoot strike is used and that, at MinRot, the rearfoot segment is flat on the ground. Since this hypothesis is not general enough (i.e., it does not consider all the possible foot strike patterns), the subsequent phases (ii) and (iii) aims to remove the drift further.

\section{Modified strap-down integration}

First, we set the quaternion ${ }_{F F}^{G F} \hat{q}(t)$ to transform the IMU $3 \mathrm{D}$ kinematics from the $\mathrm{FF}_{\text {rear }}$ into the GF. The $x$-axis of the GF was parallel to the longitudinal axis of the treadmill's belt, the $z$-axis to the lateral axis, and the $y$-axis was perpendicular to the ground surface, pointing upward (Figure 1). Typically, strap-down integration is computed between time-points at which the orientation of the $\mathrm{FF}_{\text {rear }}$ in the GF can be estimated. In walking, short zero-velocity periods during foot-flat are often used to reset the integration drift (Sabatini, 2005). As these static periods were not observed during running, we implemented a new integration method that relies on a quasi-zero velocity update at MinRot and a bidirectional strap-down integration. This method merges the strap-down integration results calculated in a forward and backward direction, awarding higher weight to the estimation originating from the closest MinRot. So, for each stride $i \in[2, N-1]$, $\mathrm{N}$ is the total number of strides, we performed strap-down integration in two directions. The quaternion ${ }_{F F}^{G F} \hat{q}_{\text {forward, } i}(t)$ with $t \in[\operatorname{MinRot}(\mathrm{i}), \operatorname{MinRot}(\mathrm{i}+1)]$ results from the forward strap-down integration and the quaternion ${ }_{F F}^{G F} \hat{q}_{\text {backward, } i}(t)$, with $t \in[\operatorname{MinRot}(\mathrm{i}-1)$, MinRot(i)], from the backward integration. Note that, we assumed the $\mathrm{FF}_{\text {rear }}$ at $\operatorname{MinRot}\left[{ }_{F F}^{G F} \hat{q}(\operatorname{MinRot})\right]$ to be aligned with the GF. The orientation difference was obtained as in Eq. 1:

$$
{ }_{F F}^{G F} \hat{q}_{\text {diff }}(t)={ }_{F F}^{G F} \hat{q}_{\text {backward, } i+1}(t) *{ }_{F F}^{G F} \hat{q}_{\text {forward }, i}(t)^{-1}
$$

We then weighted the contribution of the "backward" and "forward" estimations in the actual orientation ${ }_{F F}^{G F} \hat{q}_{\text {diff }}(t)$ through the correction of the helical angle $[\alpha(t)]$ obtained by the transformation from the quaternion notation to the axis-angle notation (quat2helic):

$$
\begin{aligned}
& (\vec{u}(t), \alpha(t))=\text { quat2helic }\left(\stackrel{G F}{F F} \hat{q}_{\text {diff }}(t)\right) \\
& \alpha_{w}(t)=\alpha(t) * \frac{t-\operatorname{MinRot}(i)}{|t-\operatorname{MinRot}(i+1)|}
\end{aligned}
$$

The corrected helical angle $\alpha_{w}(t)$ and vector $\vec{u}(t)$ were then transformed back into quaternion notation (helic2quat) and used to estimate the weighted orientation difference:

$$
{ }_{F F}^{G F} \hat{q}_{\text {diff, } w}(t)=\text { helic2quat }\left(\vec{u}(t), \alpha_{w}(t)\right)
$$

Finally, we found the rearfoot orientation as:

$$
\underset{F F}{G F} \hat{q}(t)={ }_{F F}^{G F} \hat{q}_{\text {diff,w }}(t) *{ }_{F F}^{G F} \hat{q}_{\text {forward }, i}(t)
$$

Since the forward and backward orientation estimations are linearly weighted (Eq. 3), this technique does not have jumps in the final orientation estimate ${ }_{F F}^{G F} \hat{q}(t)$.

\section{Drift modeling based on joint center acceleration}

During the stance phase of running, the kinematics of the rearfoot and forefoot segments vary upon the landing technique. The forefoot segment always has a short flat period, independently of the foot strike pattern (i.e., rearfoot strike, midfoot strike, or forefoot strike), while the rearfoot segment is usually flat only for rearfoot strikes. However, all runners have the forefoot segment that remains flat on the ground shortly after toe-strike and toward most of the pushing phase (Cavanagh and Lafortune, 1980; De Cock et al., 2005). The previously calculated rearfoot orientation ${ }_{F F}^{G F} \hat{q}(t)$ could, therefore, be incorrect due to this potential absence of the rearfoot-flat period. By modeling the foot with two segments, one can estimate the acceleration at their joint center (i.e., point $c$ in Figure 1) based on the rearfoot $\left[\vec{a}_{c, p}(t)\right]$ and forefoot $\left[\vec{a}_{c, q}(t)\right]$ accelerations. The above can be done using the function $\varphi(\vec{a}, \vec{\omega}, \vec{r})$ which shifts the acceleration $\vec{a}(t)$ of any point of a segment to another point of the same segment based on 
the segment's angular velocity $\vec{\omega}(t)$ and the translation between the two points $\vec{r}$ :

$$
\varphi(\vec{a}(t), \vec{\omega}(t), \vec{r})=\vec{a}(t)+\overrightarrow{\dot{\omega}}(t) \times \vec{r}+\vec{\omega}(t) \times(\vec{\omega}(t) \times \vec{r})
$$

The drift model in this study assumes that, during the forefootflat period, $\vec{a}_{c, p}(t)-\vec{a}_{c, q}(t)=\overrightarrow{0}$. Consequently, the orientation difference of the joint center accelerations (JCAs) $[\delta(t)]$ should also be zero or minimal. During forefoot-flat, $\vec{a}_{c, q}(t)$ can be estimated from Eq. 6 by assuming no angular rotation:

$$
\vec{a}_{c, q}(t)=\vec{a}_{q}(t)=\vec{g}+\vec{a}_{\text {treadmill }}(t)
$$

where $\vec{g}$ is the earth gravitational acceleration and $\vec{a}_{\text {treadmill }}(t)$ the acceleration of the treadmill. Note that, even if the treadmill velocity was set constant, the shearing forces acting on the belt, shortly after landing, change the speed of the treadmill, hence generating a non-zero acceleration $\vec{a}_{\text {treadmill }}(t)$. The model also assumes that each point on the rearfoot segment has a trajectory which lies on the surface of a sphere during forefoot-flat; hence, Eq. 8 describes the accelerations acting at point $\mathrm{p}$ :

$$
\vec{a}_{p}(t)=\vec{a}_{p, \text { tang }}(t)+\vec{a}_{p, \text { cent }}(t)+\vec{g}+\vec{a}_{\text {treadmill }}(t)
$$

Where, $\vec{a}_{p \text {,tang }}(t)$ is the tangential and $\vec{a}_{p \text {,cent }}(t)$ the centripetal acceleration at point $p$.

\section{Drift estimation and reduction}

To estimate the orientation drift $\delta(\mathrm{t})$, the accelerations $\vec{a}_{c, p}(t)$ and $\vec{a}_{c, q}(t)$ were calculated based on the acceleration and angular velocity at point $\mathrm{p}$ and $\mathrm{q}$, respectively. As the exact position $(p)$ of the IMU is unknown (i.e., somewhere on the dorsum of the foot), we designed a two-step optimization process to find the $\vec{r}_{p c}$ vector, necessary to find $\vec{a}_{c, p}(t)$. In the first step, the point $c^{\prime}$ is selected as the candidate position, which minimizes the norm of the tangential and centripetal accelerations. This point $c^{\prime}$ is chosen among all the rearfoot points $j$ for which $r_{p j, x}<30 \mathrm{~cm}$, $r_{p j, y}<10 \mathrm{~cm}$ and $r_{p j, z}<5 \mathrm{~cm}$ in $\mathrm{FF}_{\text {rear }}$. We used Eq. 6 to find an estimate of $\vec{r}_{p c}$, namely $\vec{r}_{p c^{\prime}}$, which minimizes Eq. 9 after removing the contribution of $\vec{g}$ (inclination) from the acceleration at point $j$ :

$$
\vec{r}_{p c^{\prime}}=\underset{\vec{r}_{p j}}{\operatorname{argmin}}\left(\left\|\varphi\left(\vec{a}_{p}(t)-{ }_{F F}^{G F} \hat{q}(t)^{-1} * G F \vec{g}, \vec{\omega}_{p}(t), \vec{r}_{p j}\right)\right\|\right),
$$$$
t \in S
$$

The function argmin returns the $\vec{r}_{p j}$ vector at which the input function is minimized. $S$ is the set of samples within the pushing phase of each stride $i$, defined as $t \in[\operatorname{MinRot}(i), T C(i)]$. During the pushing phase, the tangential and centripetal accelerations are maximum. This high signal-to-noise ratio optimizes the outcome of the minimalization function in Eq. 9. As a result, Eq. 6 and $\vec{r}_{p c^{\prime}}$ can be used to estimate the acceleration at the point $c^{\prime}$ :

$$
\vec{a}_{c^{\prime}, p}(t)=\varphi\left(\vec{a}_{p}(t), \vec{\omega}_{p}(t), \vec{r}_{p c^{\prime}}\right)
$$

If the point $c^{\prime}$ is reasonably close to the joint center $c$, the tangential and centripetal accelerations should approximately be null and, based on Eq. 7, $\vec{a}_{c^{\prime}, p}(t)-\vec{g}$ can be used as an estimate of $\vec{a}_{\text {treadmill }}(t)$.

$$
\begin{gathered}
\vec{a}_{c^{\prime}, p}(t) \cong \vec{a}_{c, q}(t)=\vec{g}+\vec{a}_{\text {treadmill }}(t) \\
\vec{a}_{c^{\prime}, p}(t)-\vec{g} \cong \vec{a}_{\text {treadmill }}(t)
\end{gathered}
$$

In the second step of optimization, $\vec{a}_{c^{\prime}, p}(t)$ was used as in Eq. 9 to refine the estimate of $\vec{r}_{p c}$ :

$$
\begin{gathered}
\vec{r}_{p c}=\underset{\vec{r}_{p x}}{\operatorname{argmin}}\left\|\varphi\left(\vec{a}_{p}(t)-\vec{a}_{c^{\prime}, p}(t), \vec{\omega}_{p}(t), \vec{r}_{p x}\right)\right\| \\
\vec{a}_{c, p}(t)=\varphi\left(\vec{a}_{p}(t), \vec{\omega}_{p}(t), \vec{r}_{p c}\right)
\end{gathered}
$$

Using Eq. 7 and Eq. 14 in GF, the orientation drift $[\delta(t)]$ was estimated, for each step, as the orientation difference between $\vec{a}_{c, p}(t)$ and $\vec{a}_{c, q}(t)$ :

$$
\delta(\mathrm{t})=\left[\cos \left(\frac{\beta(t)}{2}\right), \sin \left(\frac{\beta(t)}{2}\right) * v(t)\right]
$$

where $v(t)$ is a unit vector perpendicular to $\vec{a}_{c, p}(t)$ and $\vec{a}_{c, q}(t)$ and $\beta(t)$ is the rotation around $v(t)$ :

$$
\begin{gathered}
\beta(t)=\operatorname{acos}\left(\frac{\vec{a}_{c, q}(t) * \vec{a}_{c, p}(t)}{\left|\vec{a}_{c, q}(t)\right| *\left|\vec{a}_{c, p}(t)\right|}\right) \\
v(t)=\frac{\vec{a}_{c, q}(t) \times \vec{a}_{c, p}(t)}{\left|\vec{a}_{c, q}(t) \times \vec{a}_{c, p}(t)\right|}
\end{gathered}
$$

The orientation drift of the ith stance phase, namely $\delta_{i}$, was defined as the average quaternion (Markley et al., 2007) of $\delta(t)$ where $\mathrm{t} \in\left[\mathrm{t}_{m}-\varepsilon, \mathrm{t}_{m}+\varepsilon\right]$. The parameter $\mathrm{t}_{m}$ was found as in Eq. 18 and $\varepsilon=5 \mathrm{~ms}$.

$$
\begin{gathered}
t_{m}=\min _{t}\left(\left\|\vec{a}_{c, p}(t)\right\|-1\right) \\
\delta_{i}=\operatorname{mean}(\delta(t)), t \in\left[t_{m}-\varepsilon, t_{m}+\varepsilon\right]
\end{gathered}
$$

We then estimated the rearfoot orientation based on Eq. 5 and obtained the drift correction with Eq. 20:

$$
\begin{gathered}
\underset{F F}{G F} q\left(t_{m}\right)={ }_{F F}^{G F} \hat{q}\left(t_{m}\right) * k * \delta_{i} \\
k=1 /\left(1+e^{100 *\left(\left\|\vec{a}_{c, p}\left(t_{m}\right)\right\|-1.1\right)}\right)
\end{gathered}
$$

Note that the sigmoid function in Eq. 21 aims to reduce the weight of the update the further the norm of $\vec{a}_{c, p}\left(t_{m}\right)$ is from the unit norm. The parameters of the sigmoid function were selected such that a $10 \%$ error corresponds to a coefficient $\mathrm{k}$ equal to 0.5 . Finally, we used the same process as the MSDI method to correct the estimate of ${ }_{F F}^{G F} \hat{q}(t)$ : for each stance phase a new ${ }_{F F}^{G F} \hat{q}_{\text {forward, } i}$ and ${ }_{F F}^{G F} \hat{q}_{\text {backward,i }}$ was computed, with the center time $\mathrm{t}_{m, i}$ and with an initial orientation defined as in Eq. 19.

\section{Reference System \\ Temporal Events Detection}

We used an instrumented treadmill (T-170-FMT, Arsalis, Belgium) as a reference system for temporal events detection. The force plate (FP) recorded the 3D ground reaction forces (GRF) at 
$1000 \mathrm{~Hz}$, and a $5 \mathrm{~V}$ analog trigger synchronized the system with the IMUs. To reduce the noise on the vertical GRF signal due to the treadmill's vibration, we first applied a 2nd-order stopband Butterworth filter with edge frequencies set to 25 and $65 \mathrm{~Hz}$. Finally, the initial and terminal contact (TC) events were found using a threshold on the vertical GRF set at $7 \%$ of the participant's body weight (Falbriard et al., 2018).

\section{The 3D Orientation of the Foot With Stereophotogrammetry}

Motion tracking of the lower limbs was achieved using eight motion cameras (BTS Smart 400, BTS Bioengineering, United States) and 21 reflective markers placed on body landmarks. The system operated at $100 \mathrm{~Hz}$ and was synchronized with the IMUs and the FPusing an analog trigger (i.e., $5 \mathrm{~V}$ pulse trigger recorded on all the systems). We defined the GF of the system using three reflective markers on the horizontal plane of the treadmill; the GF $x$-axis set parallel to the belt (i.e., in the running direction) and the $z$-axis laterally to the belt, and the $y$-axis perpendicular to the $x$ and $z$ axis (Figure 1).

\section{Calibration in standing posture}

The malleolus markers (Figure 2, m5-6) were frequently torn off while running, so we recorded a 5-s standing posture at the beginning of each session. The calibration phase aimed to obtain the matrix ${ }_{T F}^{F F} R_{\text {calib }}$ which transforms the vector space from the TF to the FF. The position of the markers of the shoe remained unchanged throughout a session, so the matrix ${ }_{T F}^{F F} R_{\text {calib }}$ was considered constant and was used to process the running trials. The TF was defined using the mean position of 4 reflective markers firmly fixed around the IMU (Figure 2, m1-4) and the matrix ${ }_{T F}^{G F} R_{\text {calib }}$ was set to transform the vector space from the TF to the GF (Eqs. 22 and 23). In Eq. 22, the $\mathcal{N}$ symbol represents the normalization function, and the circumflex indicates that the average position of the marker was considered.

$$
\begin{gathered}
\overrightarrow{\tilde{x}}_{T F}=\mathcal{N}\left(\overrightarrow{\hat{m}}_{2}-\overrightarrow{\hat{m}}_{4}\right), \vec{z}_{T F}=\mathcal{N}\left(\overrightarrow{\hat{m}}_{3}-\overrightarrow{\hat{m}}_{4}\right), \\
\vec{y}_{T F}=\mathcal{N}\left(\vec{z}_{T F} \times \overrightarrow{\tilde{x}}_{T F}\right), \vec{x}_{T F}=\vec{y}_{T F} \times \vec{z}_{T F} \\
{ }_{T F}^{G F} R_{\text {calib }}=\left[\vec{x}_{T F}, \vec{y}_{T F}, \vec{z}_{T F}\right]
\end{gathered}
$$

We defined the orientation of the FF $\left({ }_{F F}^{G F} R_{\text {calib }}\right)$ using the two malleolus markers (m5-6) and the GF vertical axis, as shown in Eqs. 24 and 25.

$$
\begin{gathered}
\overrightarrow{\vec{z}}_{F F}=\mathcal{N}\left(\overrightarrow{\hat{m}}_{8}-\overrightarrow{\hat{m}}_{9}\right), \vec{y}_{F F}=[0,1,0], \\
\vec{x}_{F F}=\mathcal{N}\left(\vec{y}_{F F} \times \overrightarrow{\tilde{z}}_{F F}\right), \vec{z}_{F F}=\vec{x}_{F F} \times \vec{y}_{F F} \\
{ }_{F F}^{G F} R_{\text {calib }}=\left[\vec{x}_{F F}, \vec{y}_{F F}, \vec{z}_{F F}\right]
\end{gathered}
$$

Finally, we obtained the matrix ${ }_{T F}^{F F} R_{\text {calib }}$ using the two calibration matrices from Eq. 23 and Eq. 25.

$$
{ }_{T F}^{F F} R_{\text {calib }}={ }_{F F}^{G F} R_{\text {calib }}^{\prime} *{ }_{T F}^{G F} R_{\text {calib }}
$$

\section{Reference orientation during running}

During the running trials, only the markers $\mathrm{m} 1$ to $\mathrm{m} 4$ were kept. We calculated the TF of the foot as in Eq. 22 except that the markers' position at each time $t$ was considered and not their average position as for the calibration trials.

$$
{ }_{T F}^{G F} R(t)=\left[\vec{x}_{T F}(t), \vec{y}_{T F}(t), \vec{z}_{T F}(t)\right]
$$

Finally, we transformed the TF into the FF using the matrix from the calibration trial.

$$
{ }_{F F}^{G F} R(t)=\left({ }_{T F}^{F F} R_{\text {calib }} *{ }_{T F}^{G F} R(t)^{\prime}\right)^{\prime}={ }_{T F}^{G F} R(t) *{ }_{T F}^{F F} R_{\text {calib }}^{\prime}
$$

By definition, the columns of $\underset{F F}{G F} R(t)$ correspond to the coordinates of the TF basis vectors in the GF. The TF was computed based on the markers affixed on the IMU and was, therefore, subject to fixation artifact. Three additional markers were placed on the subtalar region as duplicates in case of unsatisfactory data quality. These markers were fixed on the shoe but suffered from recurrent marker loss as the marker on the medial side was frequently hit by the opposite foot during running. When present, however, these markers were used to visually assess the sensor-to-foot motion (i.e., wobbling of the sensor) with an average RMS difference of $3.68^{\circ}$ obtained after the low-pass filtering of the pitch angle.

\section{Validated Angles}

We calculate two reference angles using the $3 \mathrm{D}$ orientation of the foot measured by stereophotogrammetry $\left[{ }_{F F}^{G F} R(t)\right]$ : the pitch angle $\left(\theta_{\text {ref }}\right)$, defined as the projection of the FF $x$-axis onto the sagittal plane in the GF and the roll angle $\left(\rho_{\text {ref }}\right)$, defined as the projection of FF $z$-axis onto the frontal plane in the GF. These angles were also computed for the IMU system using the MSDI $\left[{ }_{F F}^{G F} \hat{q}(t)\right]$ method $\left(\theta_{\text {MSDI }}, \rho_{\text {MSDI }}\right)$ and the JCA $\left[{ }_{F F}^{G F} q(t)\right]$ method $\left(\theta_{\text {JCA }}, \rho_{\text {JCA }}\right)$. By definition, the pitch angle is zero when the rear foot remains flat on the ground and is positive when the forefoot segment is higher than the rearfoot segment. The root mean square error (RMSE) of $\theta_{\text {ref }}$ (stance) $-\theta_{\text {MSDI }}$ (stance) and $\theta_{\text {ref }}($ stance $)-\theta_{\text {JCA }}$ (stance) was estimated for each stance phase. In addition, the value of the pitch angle at initial contact (IC), i.e., the foot strike angle $\left[\theta_{\text {ref }}(\mathrm{IC}), \theta_{\mathrm{MSDI}}(\mathrm{IC})\right.$, and $\left.\theta_{\mathrm{JCA}}(\mathrm{IC})\right]$, at terminal contact (TC), i.e., the pushing angle $\left[\theta_{\text {ref }}(\mathrm{TC}), \theta_{\mathrm{MSDI}}(\mathrm{TC})\right.$, and $\left.\theta_{\mathrm{JCA}}(\mathrm{TC})\right]$ and at mean-stance $\left[\theta_{\text {ref }}(\mathrm{MS}), \theta_{\mathrm{MSDI}}(\mathrm{MS})\right.$, and $\left.\theta_{\text {JCA }}(\mathrm{MS})\right]$ were extracted from the different methods. These parameters rest on the detection accuracy of the IC and TC, so they were computed based on the results of both the FP (i.e., reference system) and the IMU (Falbriard et al., 2018). Note that, because of the potential detection error of the IMU-based method, we used the mean angle within an 8-millisecond window (i.e., \pm 2 samples at $500 \mathrm{~Hz}$ ) instead of the exact angle at IC, MS, and TC. Moreover, the time (AC) and value of the pitch angle last local maximum before IC was extracted and defined as the preactivation pitch angle $\left[\theta_{\text {ref }}(\mathrm{AC}), \theta_{\mathrm{MSDI}}(\mathrm{AC})\right.$, and $\left.\theta_{\mathrm{JCA}}(\mathrm{AC})\right]$. This feature describes the orientation of the foot shortly before landing when muscle pre-activation occurs (Kyröläinen et al., 2005). Also, as the range of the roll angle was small, therefore potentially suffering from low signal-to-noise ratio, only the activation roll angle $\left[\rho_{\text {ref }}(\mathrm{AC}), \rho_{\mathrm{MSDI}}(\mathrm{AC})\right.$, and $\left.\rho_{\mathrm{JCA}}(\mathrm{AC})\right]$ was defined as the last local minimum before IC. A negative roll angle corresponds to an inversion of the foot and a positive angle to an eversion. 


\section{A}

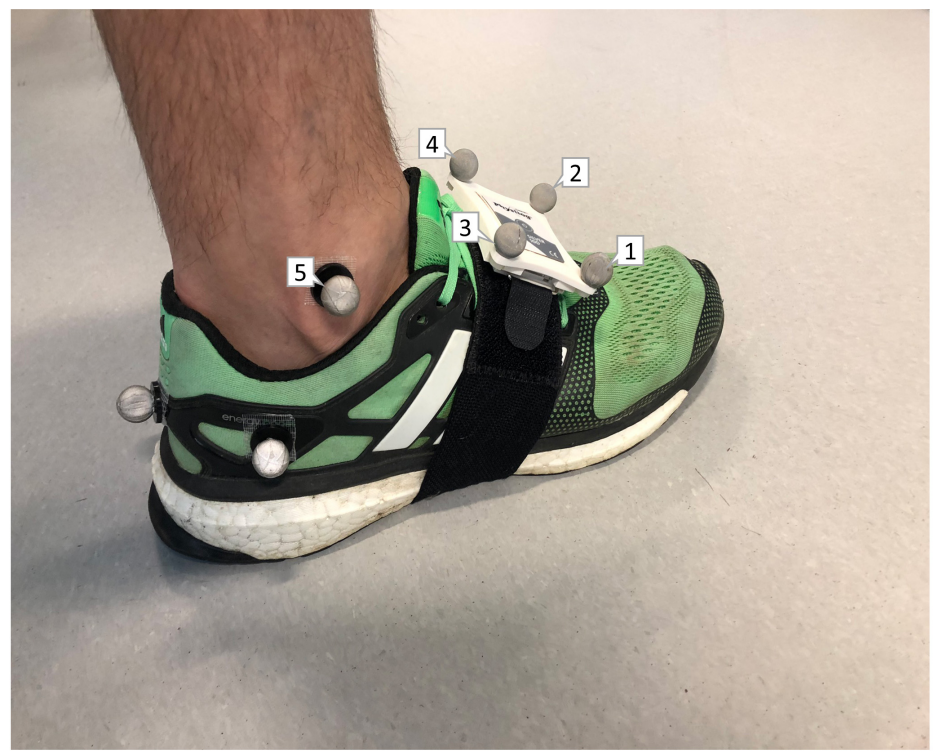

B

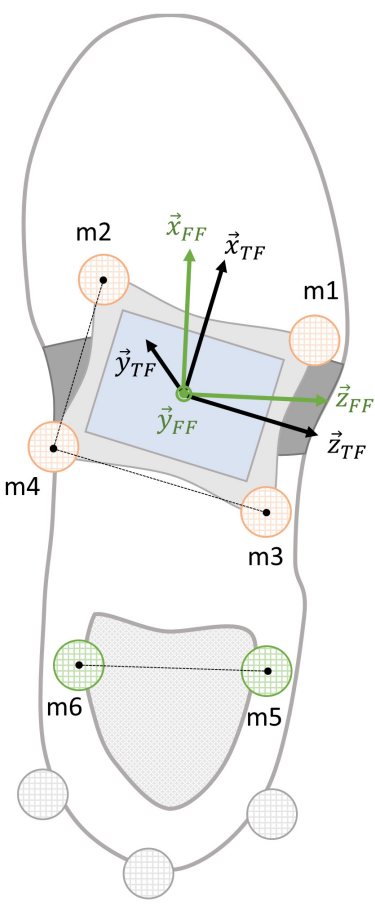

FIGURE 2 | (A) Rear/lateral view of the markers' configuration used in this study. (B) Top scheme of the markers' configuration required in the definition of the foot's technical (TF) and functional (FF) frames. Markers illustrated in orange are the one needed to set the TF, in green the FF, and in gray the duplicates which were not used in this study. Also, note that markers 5 and 6 were kept only during the calibration trials.

\section{Statistical Analysis and Error Computation}

This study focused on the trials at 8,12 , and $16 \mathrm{~km} / \mathrm{h}$, which corresponds to slow, moderate, and fast running. Trials were either removed because of instrumentation errors, protocol errors, or marker loss. We also removed the outliers steps from the data set according to the following criteria: $\theta_{\text {ref }}(M S)>10$ and $\theta_{\text {ref }}(\mathrm{AC})<-80$. After the outliers were removed, trials with less than five strides were dropped from the study.

To evaluate the error of the IMU estimations against the reference motion tracking system, we computed four statistics on the entire data set for each parameter. The bias (intra-trial mean) and precision (intra-trial STD) were computed on the strides from the same foot and the same trial. We considered the feet independently as runners may use different patterns for the left and right foot. The bias and precision were later combined among all the trials: $b_{\mu}$ the inter-trials median of the bias, $b_{\sigma}$ the inter-trials IQR of the bias, $\sigma_{\mu}$ the inter-trials median of the precision and $\sigma_{\sigma}$ the inter-trials IQR of the precision. We used the median and IQR statistics because the biases and precisions were not normally distributed.

The influence of the running speed on the intra-trial biases and precision values was tested using the non-parametric KruskalWallis test with a significance level set at $p<0.1$. This test was preferred to an ANOVA analysis because of the low number of trials and the lack of prior knowledge about the seemingly not normal distributions. Also, boxplots were used to visualize the biases and precision differences among the running speeds.
Finally, we graphically assessed the agreement between the IMUbased system and the reference motion capture system using Bland-Altman plots (Bland and Altman, 1986).

\section{RESULTS}

In total, 4252 steps were analyzed in this study. The mean \pm STD (min, max) number of recorded strides per trial was $36 \pm 6$ $(10,45)$ for a total of 59 trials $(23$ at $8 \mathrm{~km} / \mathrm{h}, 23$ at $12 \mathrm{~km} / \mathrm{h}$, and 13 at $16 \mathrm{~km} / \mathrm{h}$ ).

The pitch angles during the stance phase and obtained from the different estimation methods are shown for a rearfoot (left) and a forefoot (right) striker in Figure 3. We emphasized on the IC and TC detection differences between the FP and the IMUbased method using vertical dashed lines.

Table 1 shows the results of the inter-trials error statistics for the MSDI and JCA orientation estimation methods. The range (95\% interval) observed on the reference system for the pitch and roll angles are: $\theta_{\text {ref }}(\mathrm{IC})(-12.5,18.2), \theta_{\text {ref }}(\mathrm{MS})$ $(-11.8,-1.2), \theta_{\text {ref }}(\mathrm{TC})(-68.9,-41.6), \theta_{\text {ref }}(\mathrm{AC})(-7.8,28.9)$, and $\rho_{\text {ref }}(\mathrm{AC})(-29.5,-7.6)$. The error statistics are expressed in degrees and are shown for two different temporal events detection systems: the IMUs and the FP. When the steps are gathered regardless of their trial and using the IMU-based event detection, the mean \pm STD error $\left(^{\circ}\right)$ of the JCA method are: $\theta_{\mathrm{JCA}}(\mathrm{IC})(0.8 \pm 5.9), \theta_{\mathrm{JCA}}(\mathrm{MS})(0.2 \pm 4.7), \theta_{\mathrm{JCA}}(\mathrm{TC})(17.0 \pm 9.0)$, $\theta_{\mathrm{JCA}}(\mathrm{AC})(2.1 \pm 5.5)$, and $\rho_{\mathrm{JCA}}(\mathrm{AC})(3.1 \pm 4.9)$. Similarly, for 

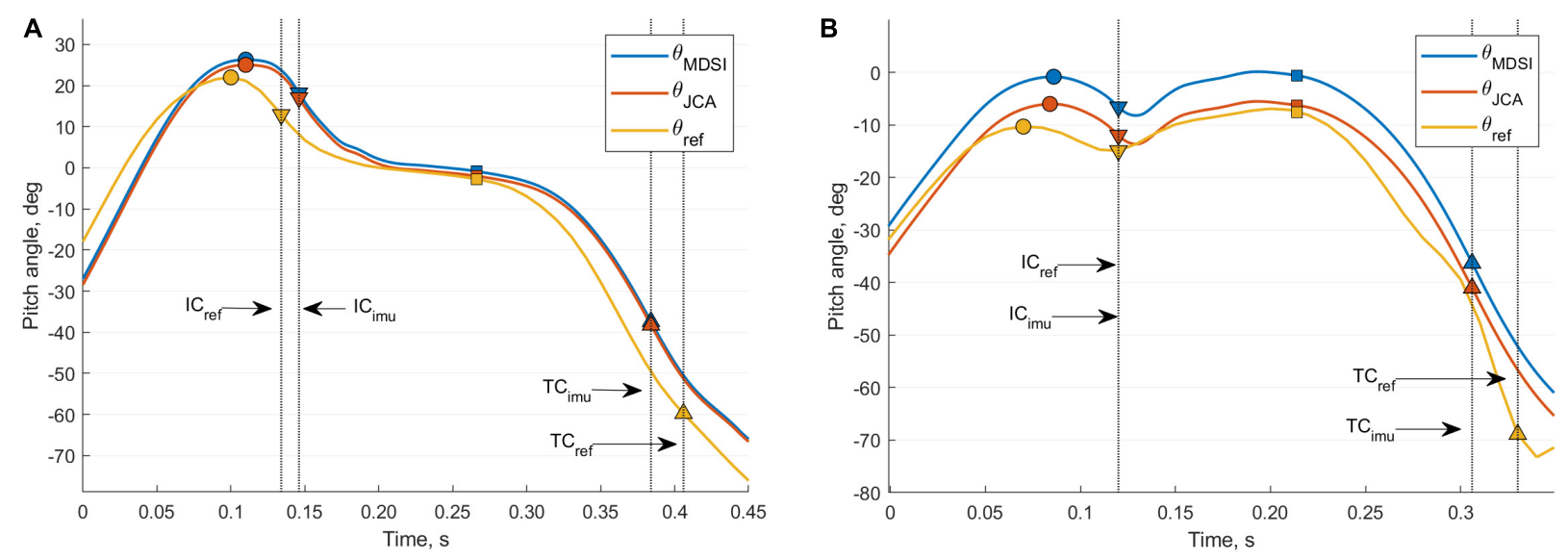

FIGURE 3 | Comparison of the pitch angle measured from different measurement systems for a rearfoot (A) and forefoot (B) striker. The blue curve is the estimation from the IMU-based MSDI method ( $\theta_{\mathrm{MSD}}$ ), the orange curve from the IMU-based JCA method ( $\left.\theta_{J C A}\right)$, and the yellow curve from the reference motion tracking system $\left(\theta_{\text {ref }}\right)$. The IC events are shown using down-pointing triangles, TC events with up-pointing triangles, MS events with squares, and the AC peaks using circles. The black vertical dashed lines accentuate the detection differences, for the IC and TC events, between the IMU and the FP system.

the MSDI method: $\theta_{\mathrm{MSDI}}(\mathrm{IC})(3.9 \pm 5.7), \theta_{\mathrm{MSDI}}(\mathrm{MS})(3.6 \pm 3.9)$, $\theta_{\mathrm{MSDI}}(\mathrm{TC})(20.2 \pm 8.8), \theta_{\mathrm{MSDI}}(\mathrm{AC})(5.3 \pm 5.2)$, and $\rho_{\mathrm{MSDI}}(\mathrm{AC})$ $(4.6 \pm 4.9)$.

Figure 4 illustrates the intra-trial biases (b) and precision $(\sigma)$ statistics obtained for the $\theta_{\mathrm{JCA}}(\mathrm{AC})$ parameter at 8,12 , and $16 \mathrm{~km} / \mathrm{h}$. Two trials at $8 \mathrm{~km} / \mathrm{h}$ had large biases $\left(23.2^{\circ}\right.$ and $\left.-14.8^{\circ}\right)$ and were cut-off from the graph for the sake of illustration. The results from the Kruskal-Wallis test suggest that the biases $(b)$ and precision $(\sigma)$ values for $\theta_{\mathrm{JCA}}(\mathrm{AC})$ and $\theta_{\mathrm{JCA}}(\mathrm{MS})$ are not affected by the running speed. However, for the parameters $\theta_{\mathrm{JCA}}(\mathrm{IC})$, $\theta_{\mathrm{JCA}}(\mathrm{TC})$ and $\rho_{\mathrm{JCA}}(\mathrm{AC})$ the precision $(\sigma)$ of the system was significantly affected $(p<0.05)$ but the intra-trial biases (b) were not $(p=0.11, p=0.21, p=0.42)$.

Finally, a Bland-Altman plot (Figure 5) shows the agreement between the IMU-based system and the reference motion capture system, with the mean (2.1) \pm STD (5.2) of the error displayed with yellow horizontal lines.

\section{DISCUSSION}

In this study, we proposed a new method to estimate the foot orientation during running, based on a single IMU on the rear foot. While IMU-based estimation generates drift due to strapdown integration operation, we proposed a MSDI supplemented with a drift compensation method (JCA). The technique relies on the assumption of a flat period in the forefoot, which is accurate for all landing strike pattern. Assuming dynamic rearfoot kinematics during the pushing phase and a static period on the forefoot segment, a two-segment biomechanical model of the foot is, therefore, well suited for running. Also, the system requires no prior knowledge about the sensor's location. The IMU must be fixed on rearfoot, and the functional calibration is automatically performed. Validated against gold standard stereophotogrammetry system, the proposed drift correction method allowed to estimate the foot orientation at mean stance phase with a bias of $0.4 \pm 3.8^{\circ}$ and precision of $3.0 \pm 1.8^{\circ}$. Note that the validation was restricted to the stance phase due to the occlusion of the markers' position during the swing phase.

In this study, the drift correction of the MSDI method is hypothetical and enforced. An implication of this is the possibility that the biases $\left(b_{\mu}\right.$ and $\left.b_{\sigma}\right)$ observed for this method cannot be extrapolated to other populations or other running conditions (e.g., various speed and style); we would certainly expect more significant biases in the case of forefoot strikers (Figure 3B). Instead, the JCA method uses a real measure of the orientation drift and correctly estimated the pitch angle around MS, while the MSDI method remained around $0^{\circ}$ (Figure 3). The RMSE analysis (Table 1) also reveals better results for the JCA method with better accuracy $\left(b_{\mu}\right.$ and $b_{\sigma}$ statistics). In contrary, the $\sigma_{\mu}$ and $\sigma_{\sigma}$ statistics suggest that the JCA method is slightly less precise. Note that these precision differences are always below 1 (Table 1) and seem reasonable given that the $\theta_{\mathrm{MSDI}}(\mathrm{MS})$ precision exposes the inter-steps variability of the participants (i.e., of the reference system). Besides, the precision of the JCA system could be improved by tuning the parameters of the sigmoid function of Eq. 21 in order to reduce the effect of outlier estimations. Also, the algorithm provides near-real-time processing of the orientation (i.e., in the order of a step), and could potentially be improved by considering the orientation of the few preceding steps in the estimation of the drift (e.g., using a weighted average).

Table 1 and Figure 3 highlight the importance of temporal events detection accuracy in the estimation of the pitch angle at IC, MS, and TC. Large errors in the measured angles result from the fact that IC and TC events are detected during phases of rapid change in pitch angle. Table 1 reveals that, when the FP system detected TC, the median bias $\left(b_{\mu}\right)$ of the JCA and the MSDI methods improved by $14.8^{\circ}$ and $16^{\circ}$, respectively. Similarly, the biases were worsened by $5.3^{\circ}$ and $6.2^{\circ}$ for IC. These findings can be explained by the fastchanging slope of the pitch angle $(\theta)$ around the IC, while it is continuously negative around TC. In consequence, the 
TABLE 1 | Inter-trial analysis of the IMU-based pitch $(\theta)$ and roll $(\rho)$ angles estimation errors with motion tracking cameras used as a reference.

\begin{tabular}{|c|c|c|c|c|c|c|c|c|}
\hline \multirow[t]{3}{*}{ Parameter } & \multicolumn{4}{|c|}{$\begin{array}{c}\text { IMU-Based } \\
\text { event detection }\end{array}$} & \multicolumn{4}{|c|}{$\begin{array}{c}\text { FP-Based } \\
\text { event detection }\end{array}$} \\
\hline & \multicolumn{2}{|l|}{$\begin{array}{c}\text { Bias } \\
\left({ }^{\circ}\right)\end{array}$} & \multicolumn{2}{|c|}{$\begin{array}{l}\text { Precision } \\
\quad\left({ }^{\circ}\right)\end{array}$} & \multicolumn{2}{|l|}{$\begin{array}{c}\text { Bias } \\
\left({ }^{\circ}\right)\end{array}$} & \multicolumn{2}{|c|}{$\begin{array}{c}\text { Precision } \\
\left({ }^{\circ}\right)\end{array}$} \\
\hline & $\mathbf{b}_{\mu}$ & $\mathbf{b}_{\sigma}$ & $\sigma_{\mu}$ & $\sigma_{\sigma}$ & $\mathbf{b}_{\mu}$ & $\mathbf{b}_{\sigma}$ & $\sigma_{\mu}$ & $\sigma_{\sigma}$ \\
\hline$\theta_{\mathrm{JCA}}(\mathrm{IC})$ & 0.9 & 6.7 & 2.4 & 1.5 & 6.2 & 6.9 & 2.2 & 1.1 \\
\hline$\theta_{\mathrm{MSDI}}(\mathrm{IC})$ & 3.7 & 6.0 & 2.1 & 1.1 & 9.9 & 7.2 & 1.8 & 0.8 \\
\hline$\theta_{\mathrm{JCA}}(\mathrm{IC})-\theta_{\mathrm{MSDI}}(\mathrm{IC})$ & -2.9 & 0.7 & 0.3 & 0.4 & -3.7 & -0.3 & 0.3 & 0.3 \\
\hline$\theta_{\mathrm{JCA}}(\mathrm{MS})$ & 0.4 & 3.8 & 3.0 & 1.8 & 1.1 & 3.5 & 1.3 & 1.1 \\
\hline$\theta_{\mathrm{MSDI}}(\mathrm{MS})$ & 3.8 & 3.8 & 2.0 & 1.7 & 4.3 & 3.1 & 0.8 & 0.4 \\
\hline$\theta_{\mathrm{JCA}}(\mathrm{MS})-\theta_{\mathrm{MSDI}}(\mathrm{MS})$ & -3.5 & 0.0 & 0.9 & 0.1 & -3.2 & 0.4 & 0.5 & 0.8 \\
\hline$\theta_{\mathrm{JCA}}(\mathrm{TC})$ & 17.4 & 8.7 & 3.2 & 1.7 & 2.6 & 8.0 & 2.8 & 1.5 \\
\hline$\theta_{\mathrm{MSDI}}(\mathrm{TC})$ & 20.8 & 7.6 & 2.8 & 1.3 & 4.8 & 7.1 & 2.4 & 1.4 \\
\hline$\theta_{\mathrm{JCA}}(\mathrm{TC})-\theta_{\mathrm{MSDI}}(\mathrm{TC})$ & -3.4 & 1.1 & 0.3 & 0.4 & -2.2 & 0.9 & 0.4 & 0.1 \\
\hline$\theta_{\mathrm{JCA}}(\mathrm{AC})$ & 2.0 & 5.9 & 1.6 & 1.1 & & & & \\
\hline$\theta_{\mathrm{MSDI}}(\mathrm{AC})$ & 5.0 & 5.7 & 1.2 & 0.9 & & & & \\
\hline$\theta_{\mathrm{JCA}}(\mathrm{AC})-\theta_{\mathrm{MSDI}}(\mathrm{AC})$ & -3.0 & 0.2 & 0.3 & 0.2 & & & & \\
\hline$\theta_{\mathrm{JCA}}($ stance $)$ & 4.5 & 2.8 & 0.8 & 0.6 & & & & \\
\hline$\theta_{\mathrm{MSDI}}($ stance $)$ & 6.2 & 4.1 & 0.6 & 0.3 & & & & \\
\hline$\theta_{\mathrm{JCA}}($ stance $)-\theta_{\mathrm{MSDI}}($ stance $)$ & -1.7 & -1.3 & 0.2 & 0.3 & & & & \\
\hline$\rho_{\mathrm{JCA}}(\mathrm{AC})$ & 3.0 & 5.7 & 1.5 & 1.3 & & & & \\
\hline$\rho_{\mathrm{MSDI}}(\mathrm{AC})$ & 4.4 & 5.5 & 1.5 & 1.2 & & & & \\
\hline$\rho_{\mathrm{JCA}}(\mathrm{AC})-\rho_{\mathrm{MSDI}}(\mathrm{AC})$ & -1.4 & 0.2 & 0.0 & 0.1 & & & & \\
\hline
\end{tabular}

The MSDI and JCA methods are evaluated in two scenarios: (1) Using temporal event detection from the IMU. (2) Using temporal event detection from the force plate (FP). The selected events are: IC, initial contact; MS, mean-stance; TC, terminal contact; $A C$, activation peak. The results of the inter-trials median $\left(b_{\mu}\right)$ and IQR $\left(b_{\sigma}\right)$ of the bias, the inter-trials median $\left(\sigma_{\mu}\right)$ and IQR $\left(\sigma_{\sigma}\right)$ of the precision and the root mean square error (RMSE) of the continuous pitch angle [ $\theta \mathrm{JCA}$ (stance), $\theta_{\mathrm{MSDI}}($ stance)] between IC and TC are presented in this table.

detection biases of IC and TC have dissimilar effects on $\theta_{\mathrm{JCA}}(\mathrm{IC}), \theta_{\mathrm{MSDI}}(\mathrm{IC}), \theta_{\mathrm{JCA}}(\mathrm{TC})$, and $\theta_{\mathrm{MSDI}}(\mathrm{TC})$. Furthermore, these estimations are sensitive to synchronization delays between the reference systems and the IMUs.

Because the AC event is not affected by the temporal events detection technique, a more detailed analysis was performed on the $\theta_{\mathrm{JCA}}(\mathrm{AC})$ parameter. The parameter $\rho_{\mathrm{JCA}}(\mathrm{AC})$ is also unaffected by the detection accuracy of the temporal events; however, the pitch angle was preferred because of the lack of generality in the roll angle drift correction hypothesis. The assumption of a null roll angle for the forefoot segment may be incorrect for subjects with pathological pronation/supination. In Figure 5, the optical motion-tracking system and the IMU-based system demonstrate a good agreement across the range of angles.

Bailey and Harle (2014) used two methods (linear de-drifting and extended Kalman filter) to compute the orientation of the foot in 5 subjects based on shoe-mounted IMUs. They reported an error (mean + STD) for $\theta(\mathrm{IC})$ of $1.92 \pm 1.09^{\circ}$ at $8.28 \mathrm{~km} / \mathrm{h}$ and $3.18 \pm 1.19^{\circ}$ at $12.24 \mathrm{~km} / \mathrm{h}$. The present JCA results $\left(0.8 \pm 5.9^{\circ}\right)$ show a better bias but a lower precision. The lower performance in precision might be associated with the higher diversity of subjects and speeds analyzed in this study. Also, the authors assumed that the pitch and roll angles were similar for
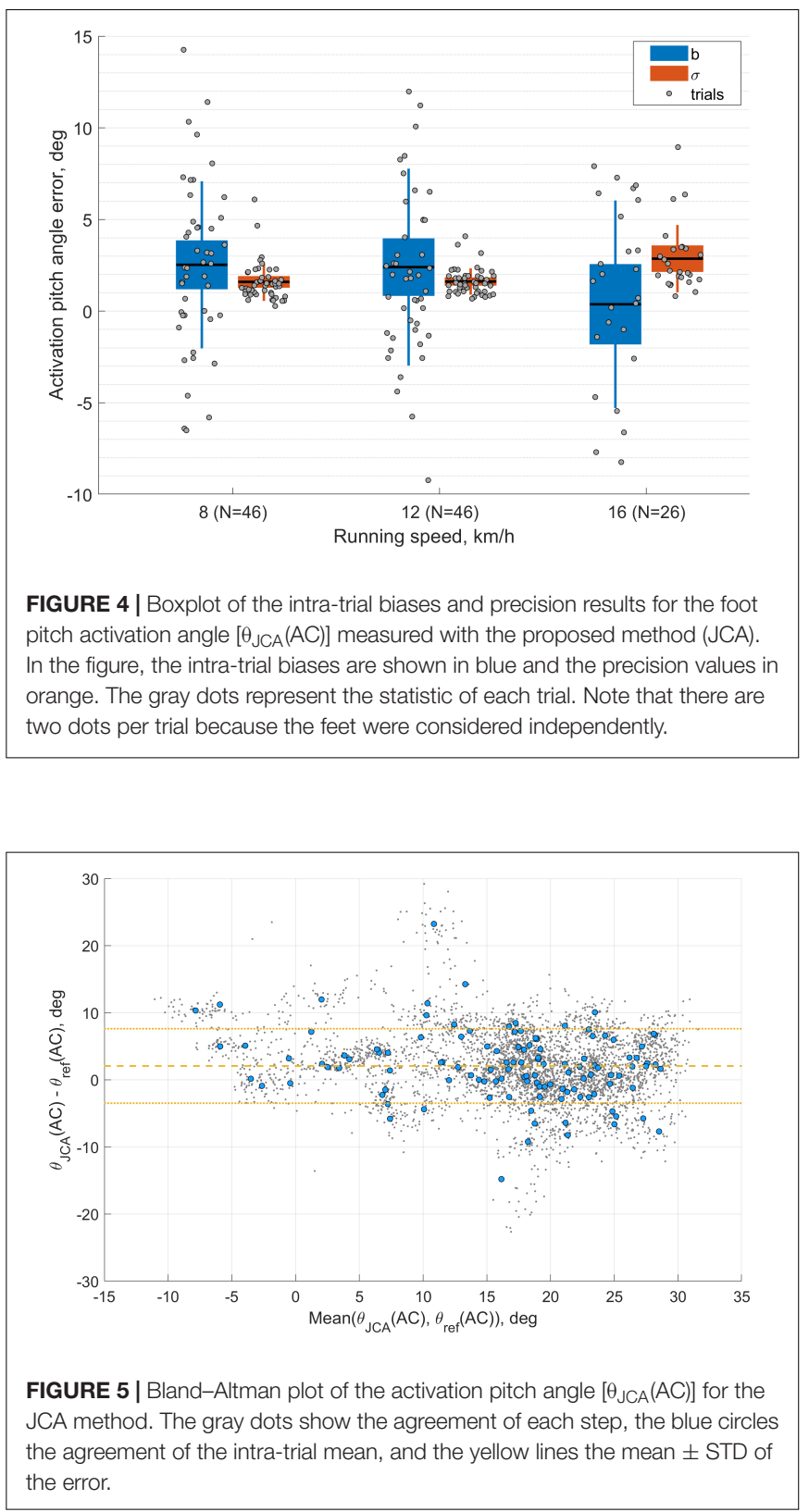

every stance phase, hence reducing the inter-steps variability of the system. Koska et al. (2018) used trapezoidal integration of gyroscopic measurements to estimate the orientation of the foot during the stance phase. The authors reported error biases $\left({ }^{\circ}\right)$ $\pm 95 \%$ limits of agreement $\left(^{\circ}\right)$ of $-3.1 \pm(-7,3.4)$ at $10 \mathrm{~km} / \mathrm{h}$, $-3.8 \pm(-7.6,2.1)$ at $12 \mathrm{~km} / \mathrm{h}$, and $-5.9 \pm(-11.1,1.8)$ at $15 \mathrm{~km} / \mathrm{h}$ in the estimation of the sagittal plane (i.e., pitch angle) range of motion during stance phase. Although heel-off events were defined using a fixed time window, their observations corroborate with the results of the MSDI method. Shiang et al. (2016) also assumed the presence of a foot-flat period (i.e., pitch angle $=0^{\circ}$ ) during stance, as for the MSDI method used in the present study, and defined the difference between two local 
maximums as the strike index. The range of angles $\left(-5^{\circ}, 27^{\circ}\right)$ reported for the strike index reflect those obtained for $\theta_{\text {JCA }}(\mathrm{AC})$ and $\theta_{\mathrm{MSDI}}(\mathrm{AC})$ parameters in this study. Also, in Altman and Davis (2012), the authors concluded that the foot strike angle, obtained from an optical motion capture system, is an acceptable measure of foot strike pattern, and proposed the following classification limits: rearfoot strike $>8^{\circ}$, midfoot strike between $-1.6^{\circ}$ and $8^{\circ}$, and forefoot strike $<1.6$. These results, with a midfoot strike classification range of $9.6^{\circ}$, suggest that the JCA method provides an acceptable measure of the pitch angle, with an accuracy of $2.0 \pm 5.9^{\circ}$ and $0.9 \pm 6.7^{\circ}$ for the $\theta_{\mathrm{JCA}}(\mathrm{AC})$ and $\theta_{\text {JCA }}(\mathrm{IC})$ parameters, respectively. However, such a conclusion does not hold for the MSDI method (Table 1). A validation study on walking analysis (Bourgeois et al., 2014) reported accuracy and precision of $0.5 \pm 2.9^{\circ}$ and $3.9 \pm 5.8^{\circ}$ in the estimation of the pitch angle $(\theta)$ at initial and TC, respectively. In comparison, we observed (when the steps were gathered regardless of their trial) a $0.8 \pm 5.9^{\circ}$ and $17.0 \pm 9.0^{\circ}$ accuracy and precision for $\theta_{\mathrm{JCA}}$ (IC) and $\theta_{\mathrm{JCA}}(\mathrm{TC})$ parameters. The lower performance may partly be explained by the lower detection accuracy of the initial and TC in running and by the highly dynamic motion of the foot in running generating a greater level of noise during the period of stance.

The results from the Kruskal-Wallis test suggest that neither the bias nor the precision of the $\theta_{\mathrm{JCA}}(\mathrm{AC})$ and $\theta_{\mathrm{JCA}}(\mathrm{MS})$ parameters were significantly affected by the running speed. However, we observed significant differences in the precision of $\theta_{\mathrm{JCA}}(\mathrm{IC})$ and $\theta_{\mathrm{JCA}}(\mathrm{TC})$. This possibly results from the effect speed has on the detection precision and accuracy of IC and TC (Falbriard et al., 2018). Note that there is a performance tradeoff made by the system and associated with the running speed. At low running speeds, the norm of the tangential and centripetal accelerations during the pushing phase is small and therefore decreases the performance of the automatic estimation of $\vec{r}_{p c}$ in Eqs. 9-13. Conversely, ground contact times are longer at low running speeds (Nummela et al., 2007), increasing the probability of sufficiently long static periods to directly estimate the $3 \mathrm{D}$ orientation of the rearfoot segment.

It is essential to bear in mind that the present study was performed on a $0^{\circ}$ inclined treadmill. Consequently, the results reported in this document cannot be generalized to uphill and downhill running. Also, we obtained the reference orientation based on markers on the sensors rather than on the shoe; therefore, the protocol constraints such as the lightweight IMU and the IMU fixation are aspects that could affect the detection results. Finally, the present study raises the possibility for the JCA method to be tested on active gait methods other than running (e.g., Nordic walking).

\section{REFERENCES}

Ahn, A. N., Brayton, C., Bhatia, T., and Martin, P. (2014). Muscle activity and kinematics of forefoot and rearfoot strike runners. J. Sport Health Sci. 3, 102-112. doi: 10.1016/j.jshs.2014.03 .007

Altman, A. R., and Davis, I. S. (2012). A kinematic method for footstrike pattern detection in barefoot and shod runners. Gait Posture 35, 298-300. doi: 10.1016/ j.gaitpost.2011.09.104

\section{CONCLUSION}

In this study, we proposed and validated a new method to estimate and correct the orientation drift estimation based on a foot-worn IMU using a two-segment model of the foot for drift removal. The validation compared sagittal and frontal plane angles obtained from an optical motion-tracking system with the estimation based on wearable inertial sensors. We showed that the pitch angle at mid-stance can be estimated with an inter-trial median \pm IQR of $0.4 \pm 3.8^{\circ}$ and an intertrial precision median $\pm \mathrm{IQR}$ of $3.0 \pm 1.8^{\circ}$. Although running speed can affect the detection performance, the system showed a good agreement with the gold standard optical motion-tracking system. Apart from the short standing period used for the functional calibration, the proposed system is fully plug-andplay. It requires no prior knowledge about the position of the sensors and needs no magnetometer.

\section{DATA AVAILABILITY STATEMENT}

The raw data supporting the conclusions of this article will be made available by the authors, without undue reservation, to any qualified researcher.

\section{ETHICS STATEMENT}

The studies involving human participants were reviewed and approved by the CCER-VD. The patients/participants provided their written informed consent to participate in this study.

\section{AUTHOR CONTRIBUTIONS}

MF, FM, BM, GM, and KA conceptualized the study design and contributed to the analysis and interpretation of the data. MF and FM conducted the data collection. MF designed the algorithms. KA supervised the study. MF drafted the manuscript, and all other authors revised it critically. All authors approved the final version and agreed to be accountable for all aspects of this work.

\section{FUNDING}

This study was supported by the Swiss CTI grant number 17664.1 PFNM-NM.

Arndt, A., Wolf, P., Liu, A., Nester, C., Stacoff, A., Jones, R., et al. (2007). Intrinsic foot kinematics measured in vivo during the stance phase of slow running. J. Biomech. 40, 2672-2678. doi: 10.1016/j.jbiomech.2006.12.009

Bailey, G. P., and Harle, R. (2014). Assessment of foot kinematics during steady state running using a foot-mounted IMU. Procedia Eng. 72, 32-37. doi: 10. 1016/j.proeng.2014.06.009

Bland, J. M., and Altman, D. (1986). Statistical methods for assessing agreement between two methods of clinical measurement. Lancet 327, 307-310. doi: $10.1016 / \mathrm{s} 0140-6736(86) 90837-8$ 
Bourgeois, A. B., Mariani, B., Aminian, K., Zambelli, P. Y., and Newman, C. J. (2014). Spatio-temporal gait analysis in children with cerebral palsy using, foot-worn inertial sensors. Gait Posture 39, 436-442. doi: 10.1016/j.gaitpost.2013.08.029

Boyer, E. R., Rooney, B. D., and Derrick, T. R. (2014). Rearfoot and midfoot or forefoot impacts in habitually shod runners. Med. Sci. Sports Exerc. 46, 1384-1391. doi: 10.1249/mss.0000000000000234

Breine, B., Malcolm, P., Frederick, E. C., and De, D. C. (2014). Relationship between running speed and initial foot contact patterns. Med. Sci. Sports Exerc. 46, 1595-1603. doi: 10.1249/MSS.0000000000000267

Camomilla, V., Bergamini, E., Fantozzi, S., and Vannozzi, G. (2018). Trends supporting the in-field use of wearable inertial sensors for sport performance evaluation: a systematic review. Sensors 18:873. doi: 10.3390/s18030873

Cavanagh, P. R., and Lafortune, M. A. (1980). Ground reaction forces in distance running. J. Biomech. 13, 397-406. doi: 10.1016/0021-9290(80)90033-0

Chew, D. K., Ngoh, K. J. H., and Gouwanda, D. (2018). Estimating running spatial and temporal parameters using an inertial sensor. Sports Eng. 21, 115-122. doi: 10.1007/s12283-017-0255-9

Daoud, A. I., Geissler, G. J., Wang, F., Saretsky, J., Daoud, Y. A., and Lieberman, D. E. (2012). Foot strike and injury rates in endurance runners: a retrospective study. Med. Sci. Sports Exerc. 44, 1325-1334. doi: 10.1249/MSS. 0b013e3182465115

de Almeida, M. O., Saragiotto, B. T., Yamato, T. P., and Lopes, A. D. (2015). Is the rearfoot pattern the most frequently foot strike pattern among recreational shod distance runners? Phys. Ther. Sport 16, 29-33. doi: 10.1016/j.ptsp.2014.02.005

De Cock, A., De Clercq, D., Willems, T., and Witvrouw, E. (2005). Temporal characteristics of foot roll-over during barefoot jogging: reference data for young adults. Gait Posture 21, 432-439. doi: 10.1016/j.gaitpost.2004.05.004

Dejnabadi, H., Jolles, B. M., Casanova, E., Fua, P., and Aminian, K. (2006). Estimation and visualization of sagittal kinematics of lower limbs orientation using body-fixed sensors. IEEE Trans. Biomed. Eng. 53, 1385-1393. doi: 10. 1109/tbme.2006.873678

Falbriard, M., Meyer, F., Mariani, B., Millet, G. P., and Aminian, K. (2018). Accurate estimation of running temporal parameters using foot-worn inertial sensors. Front. Physiol. 9:610. doi: 10.3389/fphys.2018.00610

Fasel, B., Spörri, J., Chardonnens, J., Kröll, J., Müller, E., and Aminian, K. (2018). Joint inertial sensor orientation drift reduction for highly dynamic movements. IEEE J. Biomed. Health Inform. 22, 77-86. doi: 10.1109/JBHI.2017.2659758

Favre, J., Jolles, B. M., Aissaoui, R., and Aminian, K. (2008). Ambulatory measurement of 3D knee joint angle. J. Biomech. 41, 1029-1035. doi: 10.1016/j. jbiomech.2007.12.003

Ferraris, F., Grimaldi, U., and Parvis, M. (1995). Procedure for effortless in-field calibration of three-axis rate gyros and accelerometers. Sens. Mater. 7, 311-311.

Foxlin, E. (2005). Pedestrian tracking with shoe-mounted inertial sensors. IEEE Comput. Graph. Appl. 25, 38-46. doi: 10.1109/mcg.2005.140

Giandolini, M., Poupard, T., Gimenez, P., Horvais, N., Millet, G. Y., Morin, J. B., et al. (2014). A simple field method to identify foot strike pattern during running. J. Biomech. 47, 1588-1593. doi: 10.1016/j.jbiomech.2014.03.002

Goss, D. L., and Gross, M. T. (2012). Relationships among self-reported shoe type, footstrike pattern, and injury incidence. U.S. Army Med. Dep. J. 25-30.

Gruber, A. H., Boyer, K. A., Derrick, T. R., and Hamill, J. (2014). Impact shock frequency components and attenuation in rearfoot and forefoot running. J. Sport Health Sci. 3, 113-121. doi: 10.1016/j.jbiomech.2016.04.001

Gruber, A. H., Umberger, B. R., Braun, B., and Hamill, J. (2013). Economy and rate of carbohydrate oxidation during running with rearfoot and forefoot strike patterns. J. Appl. Physiol. 115, 194-201. doi: 10.1152/japplphysiol.01437.2012

Hamill, J., and Gruber, A. H. (2017). Is changing footstrike pattern beneficial to runners? J. Sport Health Sci. 6, 146-153. doi: 10.1016/j.jshs.2017.02.004

Horvais, N., and Samozino, P. (2013). Effect of midsole geometry on foot-strike pattern and running kinematics. Footwear Sci. 5, 81-89. doi: 10.1080/19424280. 2013.767863

Kasmer, M. E., Liu, X. C., Roberts, K. G., and Valadao, J. M. (2013). Foot-strike pattern and performance in a marathon. Int. J. Sports Physiol. Perform. 8, 286-292. doi: 10.1123/ijspp.8.3.286

Koska, D., Gaudel, J., Hein, T., and Maiwald, C. (2018). Validation of an inertial measurement unit for the quantification of rearfoot kinematics during running. Gait Posture 64, 135-140. doi: 10.1016/j.gaitpost.2018.06.007

Kyröläinen, H., Avela, J., and Komi, P. V. (2005). Changes in muscle activity with increasing running speed. J. Sports Sci. 23, 1101-1109. doi: 10.1080/ 02640410400021575
Larson, P. (2014). Comparison of foot strike patterns of barefoot and minimally shod runners in a recreational road race. J. Sport Health Sci. 3, 137-142. doi: 10.1016/j.jshs.2014.03.003

Larson, P., Higgins, E., Kaminski, J., Decker, T., Preble, J., Lyons, D., et al. (2011). Foot strike patterns of recreational and sub-elite runners in a longdistance road race. J. Sports Sci. 29, 1665-1673. doi: 10.1080/02640414.2011. 610347

Lavcanska, V., Taylor, N. F., and Schache, A. G. (2005). Familiarization to treadmill running in young unimpaired adults. Hum. Mov. Sci. 24, 544-557. doi: 10.1016/ j.humov.2005.08.001

Lieberman, D. E., Venkadesan, M., Werbel, W. A., Daoud, A. I., D’andrea, S., Davis, I. S., et al. (2010). Foot strike patterns and collision forces in habitually barefoot versus shod runners. Nature 463, 531-535. doi: 10.1038/nature 08723

Lorenz, D. S., and Pontillo, M. (2012). Is there evidence to support a forefoot strike pattern in barefoot runners? Sports Health 4, 480-484. doi: 10.1177/ 1941738112448055

Mariani, B., Hoskovec, C., Rochat, S., Büla, C., Penders, J., and Aminian, K. (2010). 3D gait assessment in young and elderly subjects using foot-worn inertial sensors. J. Biomech. 43, 2999-3006. doi: 10.1016/j.jbiomech.2010. 07.003

Markley, F. L., Cheng, Y., Crassidis, J. L., and Oshman, Y. (2007). Averaging quaternions. J. Guid. Control Dyn. 30, 1193-1197. doi: 10.2514/1.28949

Meyer, C., Mohr, M., Falbriard, M., Nigg, S. R., and Nigg, B. M. (2018). Influence of footwear comfort on the variability of running kinematics. Footwear Sci. 10, 29-38. doi: 10.1080/19424280.2017.1388296

Miller, R. H., and Hamill, J. (2015). Optimal footfall patterns for cost minimization in running. J. Biomech. 48, 2858-2864. doi: 10.1016/j.jbiomech.2015.04.019

Monaghan, G. M., Hsu, W. H., Lewis, C. L., Saltzman, E., Hamill, J., and Holt, K. G. (2014). Forefoot angle at initial contact determines the amplitude of forefoot and rearfoot eversion during running. Clin. Biomech. 29, 936-942. doi: 10.1016/j.clinbiomech.2014.06.011

Muñoz-Jimenez, M., Latorre-Román, P. A., Soto-Hermoso, V. M., and GarcíaPinillos, F. (2015). Influence of shod/unshod condition and running speed on foot-strike patterns, inversion/eversion, and vertical foot rotation in endurance runners. J. Sports Sci. 33, 2035-2042. doi: 10.1080/02640414.2015.1026377

Nigg, B. M., Cole, G. K., and Nachbauer, W. (1993). Effects of arch height of the foot on angular motion of the lower extremities in running. J. Biomech. 26, 909-916. doi: 10.1016/0021-9290(93)90053-h

Nummela, A., Keränen, T., and Mikkelsson, L. O. (2007). Factors related to top running speed and economy. Int. J. Sports Med. 28, 655-661. doi: 10.1055/s2007-964896

Park, S. K., and Suh, Y. S. (2010). A zero velocity detection algorithm using inertial sensors for pedestrian navigation systems. Sensors 10, 9163-9178. doi: 10.3390/ s101009163

Perl, D. P., Daoud, A. I., and Lieberman, D. E. (2012). Effects of footwear and strike type on running economy. Med Sci Sports Exerc 44, 1335-1343. doi: 10.1249/MSS.0b013e318247989e

Perry, S. D., and Lafortune, M. A. (1995). Influences of inversion/eversion of the foot upon impact loading during locomotion. Clin. Biomech. 10, 253-257. doi: 10.1016/0268-0033(95)00006-7

Riley, P. O., Dicharry, J., Franz, J., Della Croce, U., Wilder, R. P., and Kerrigan, D. C. (2008). A kinematics and kinetic comparison of overground and treadmill running. Med. Sci. Sports Exerc. 40, 1093-1100. doi: 10.1249/MSS. 0b013e3181677530

Sabatini, A. M. (2005). Quaternion-based strap-down integration method for applications of inertial sensing to gait analysis. Med. Biol. Eng. Comput. 43, 94-101. doi: $10.1007 / \mathrm{bf} 02345128$

Sabatini, A. M., Martelloni, C., Scapellato, S., and Cavallo, F. (2005). Assessment of walking features from foot inertial sensing. IEEE Trans. Biomed. Eng. 52, 486-494. doi: 10.1109/tbme.2004.840727

Shiang, T. Y., Hsieh, T. Y., Lee, Y. S., Wu, C. C., Yu, M. C., Mei, C. H., et al. (2016). Determine the foot strike pattern using inertial sensors. J. Sens. 2016:4759626. doi: 10.3390/sports7080184

Skog, I., Handel, P., Nilsson, J. O., and Rantakokko, J. (2010). Zero-velocity detection-an algorithm evaluation. IEEE Trans. Biomed. Eng. 57, 2657-2666. doi: 10.1109/tbme.2010.2060723

Strohrmann, C., Harms, H., Tröster, G., Hensler, S., and Müller, R. (2011). “Out of the lab and into the woods: kinematic analysis in running using wearable sensors," in Proceedings of the 13th International Conference on Ubiquitous 
Computing (New York, NY: Association for Computing Machinery), 119-122.

Yong, J. R., Silder, A., and Delp, S. L. (2014). Differences in muscle activity between natural forefoot and rearfoot strikers during running. J. Biomech. 47, 3593-3597. doi: 10.1016/j.jbiomech.2014. 10.015

Yuan, Q., Asadi, E., Lu, Q., Yang, G., and Chen, I.-M. (2019). “Uncertainty-Based IMU orientation tracking algorithm for dynamic motions," in Proceedings of the IEEE/ASME Transactions on Mechatronics, 872-882.

Zhang, R., Yang, H., Höflinger, F., and Reindl, L. M. (2017). Adaptive zero velocity update based on velocity classification for pedestrian tracking. IEEE Sens. J. 17, 2137-2145. doi: 10.1109/jsen.2017. 2665678
Conflict of Interest: BM was employed by the company Gait Up.

The remaining authors declare that the research was conducted in the absence of any commercial or financial relationships that could be construed as a potential conflict of interest.

Copyright $\odot 2020$ Falbriard, Meyer, Mariani, Millet and Aminian. This is an openaccess article distributed under the terms of the Creative Commons Attribution License (CC BY). The use, distribution or reproduction in other forums is permitted, provided the original author(s) and the copyright owner(s) are credited and that the original publication in this journal is cited, in accordance with accepted academic practice. No use, distribution or reproduction is permitted which does not comply with these terms. 